\title{
Primary meningeal melanoma masquerading as neurofibromatosis type 2: illustrative case
}

\author{
Melanie Lang-Orsini, MB, BCh, BAO, ${ }^{1}$ Julian Wu, MD, ${ }^{2}$ Carl B. Heilman, MD, ${ }^{2}$ Alina Kravtsova, MD, ${ }^{3}$ Gene Weinstein, MD, ${ }^{3}$ \\ Neel Madan, $M D^{3}$ and Knarik Arkun, $\mathrm{MD}^{1,2}$ \\ Departments of ${ }^{1}$ Pathology and Laboratory Medicine, ${ }^{2}$ Neurosurgery, and ${ }^{3}$ Radiology, Tufts Medical Center, Boston, Massachusetts
}

BACKGROUND Primary meningeal melanocytic neoplasms are exceedingly rare tumors, representing only $0.06 \%$ to $0.1 \%$ of all primary brain tumors and ranging in spectrum from benign localized tumors to highly aggressive malignant lesions. The diagnosis of these tumors is often challenging from clinical, radiological, and pathologic standpoints. Equally challenging is the distinction between primary meningeal melanocytic neoplasm and metastatic melanoma.

OBSERVATIONS The authors reported the case of a 41-year-old man with imaging findings diagnostic of neurofibromatosis type 2: bilateral internal auditory canal lesions (most consistent with bilateral vestibular schwannomas), two dura-based lesions presumed to be meningiomas, multiple spinal lesions consistent with peripheral nerve sheath tumors, and one intramedullary spinal lesion consistent with an ependymoma. Biopsy of these lesions revealed melanocytic neoplasms with mild to moderate atypia and a mildly elevated proliferation index, which made the distinction between benign and malignant challenging. In addition, the disseminated nature of these tumors made it difficult to determinate whether they arose from the meninges or represented metastases from an occult primary melanoma.

LESSONS This case illustrated the challenges presented by the diagnosis of meningeal melanocytic neoplasms and highlighted the importance of integrating the clinical and radiographic findings with histologic appearance and molecular studies.

https://thejns.org/doi/abs/10.3171/CASE21444

KEYWORDS meningeal melanoma; melanocytoma; melanomatosis; melanocytosis; neurocutaneous melanosis

Primary melanocytic tumors of the central nervous system (CNS) are a group of rare neoplasms with an annual incidence of 1 per 10 million, ranging in spectrum from benign tumors to highly aggressive malignant lesions. ${ }^{1,2}$ They are thought to originate from melanocytes that reside in the leptomeninges and were first described by Virchow in 1859, when he reported the autopsy findings of a male adult with diffuse leptomeningeal melanocytosis. ${ }^{3}$ Melanocytic neoplasms present as localized lesions such as benign melanocytoma, malignant melanoma, or a neoplasm of intermediate grade, which sits somewhere in between. Diffuse melanocytic lesions that do not form macroscopic masses are termed melanocytosis if they are benign or melanomatosis if they are malignant. ${ }^{1,2}$

These tumors may present significant diagnostic difficulties from clinical, radiological, and pathologic standpoints for several reasons.
First, these tumors may mimic other lesions radiologically, such as meningioma, ependymoma, or schwannoma, and any melanotic lesion histologically, from melanocytosis to melanotic schwannoma and melanoma. Second, separation of these tumors into either benign or malignant categories based on histological findings alone may be difficult, especially in biopsy specimens with scant diagnostic material. Finally, determining whether these tumors truly represent primary CNS tumors as opposed to metastatic lesions, which are far more common, is challenging because it is primarily a diagnosis of exclusion. This distinction is important, however, because of differences in prognosis and treatment modalities between the two tumors.

We report the case of a 41-year-old man with primary meningeal melanomatosis masquerading as neurofibromatosis type 2. This case illustrates the above-mentioned clinical, radiological, and

ABBREVIATIONS CNS = central nervous system; MRI = magnetic resonance imaging; WHO $=$ World Health Organization INCLUDE WHEN CITING Published November 15, 2021; DOI: 10.3171/CASE21444.

SUBMITTED August 5, 2021. ACCEPTED August 10, 2021.

(c) 2021 The authors, CC BY-NC-ND 4.0 (http://creativecommons.org/licenses/by-nc-nd/4.0/). 
pathological diagnostic difficulties that may arise when evaluating these neoplasms. In addition, we review the recent literature concerning primary meningeal melanocytic neoplasms with a focus on diagnosis, classification, and recently described molecular features.

\section{Illustrative Case}

We report the case of a 41-year-old man with no significant past medical history who presented with a 6-month history of headaches and hearing loss and back pain with bilateral leg tingling. Magnetic resonance imaging (MRI) of the head and spine showed bilateral enhancing masses in the internal auditory canals, most consistent with bilateral vestibular schwannomas. In this context, an additional extraaxial contrast-enhancing lesion in the right Sylvian fissure adjacent to the right inferior frontal lobe $(2.0 \times 1.9 \times 1.8 \mathrm{~cm})$ and an extraaxial contrast-enhancing lesion in the anterior aspect of the right middle cranial fossa $(1.9 \times 1.6 \times 1.8 \mathrm{~cm})$ were thought to be most consistent with meningiomas. In the spine, there was a $2.8 \times 1.0 \times 2.7-\mathrm{cm}$ cystic and solid intramedullary expansile lesion at the level of $\mathrm{C} 6$ and $\mathrm{C7}$, with the solid component demonstrating homogenous enhancement, which was thought to be an ependymoma (Fig. 1). In addition, there were multiple small intradural extramedullary enhancing lesions scattered throughout the spinal canal, which were thought to be peripheral nerve sheath tumors and/or meningiomas. A clinical diagnosis of neurofibromatosis type 2 was rendered.

The patient was referred for neurosurgical evaluation and a C6C7 laminectomy with biopsy of the intramedullary and leptomeningeal lesions. Intraoperative evaluation revealed multiple pigmented lesions on the arachnoid and a pigmented lesion on the dorsal nerve root of $\mathrm{C} 6$. Pathologic examination of the cervical lesions resulted in a final diagnosis of a cellular spindle cell melanocytic lesion, the differential of which included melanotic schwannoma, melanocytoma, and melanoma (Fig. 2). The sample was sent for next-generation sequencing to aid in further classification.

The patient was referred to the dermatology and ophthalmology departments for evaluation for possible metastatic melanoma. A full skin examination revealed a pigmented lesion on the right lateral shin. Pathologic examination of a shave biopsy of this lesion revealed it to be a lentiginous junctional nevus.
Follow-up brain MRI revealed an increase in size in the frontal and temporal brain lesions, and the decision was made to resect them with the hope of obtaining additional tissue for diagnosis and molecular studies. A final diagnosis of an atypical melanocytic neoplasm, most likely representing a primary meningeal melanoma, was rendered (Fig. 3).

\section{Pathological Findings}

Gross examination of the spinal lesions revealed multiple small fragments of gray-black tissue. Histological examination of all three specimens revealed a cellular lesion consisting of sheets of pigmented spindle cells with occasional epithelioid forms. Some of the nuclei had prominent nucleoli and others had longitudinal nuclear grooves. Mitotic figures and necrosis were not seen. Immunohistochemistry showed that the lesional cells expressed MART-1 and were negative for BRAF V600E. The Ki-67 proliferation index was slightly elevated at $3 \%-5 \%$. A final diagnosis of hypercellular spindle cell melanocytic lesion with a differential diagnosis of melanotic schwannoma, melanocytoma, and melanoma was rendered.

Pathology of the resected frontal and temporal tumors showed cellular melanocytic lesions, similar to the spinal lesions, but with more atypia and numerous mitotic figures. Focal brain invasion was noted. Immunohistochemistry showed that the lesional cells expressed MART1 and were negative for BRAF V600E. The Ki-67 proliferation index was higher than that of the spinal lesions, at $5 \%-10 \%$. The rapid growth of the brain lesions over a relatively short period of time (5 months) was concerning and most in keeping with a diagnosis of a tumor with aggressive behavior. Next-generation sequencing detected mutations in GNAQ (Q209L) and SF3B1. These findings further supported the diagnosis of primary meningeal melanoma.

The case was sent to Memorial Sloan Kettering Cancer Center for consultation, and additional studies were performed. Immunohistochemical staining for PRAME revealed the tumor cells to be diffusely positive. Cytogenetic analysis detected the following chromosomal abnormalities: gains in chromosome 6 (segments 6pter-q22.3, 6p22.3-p21.1, and 6q25.3-qter), gains in chromosome 8 (segments 8q13.3-q21.3 and 8q21.3-qter, including MYC), loss in chromosome 9 (segment 9pterp22.2), and gain in chromosome 17 (segment 17q22-qter). These findings were in keeping with the malignant nature of these lesions.
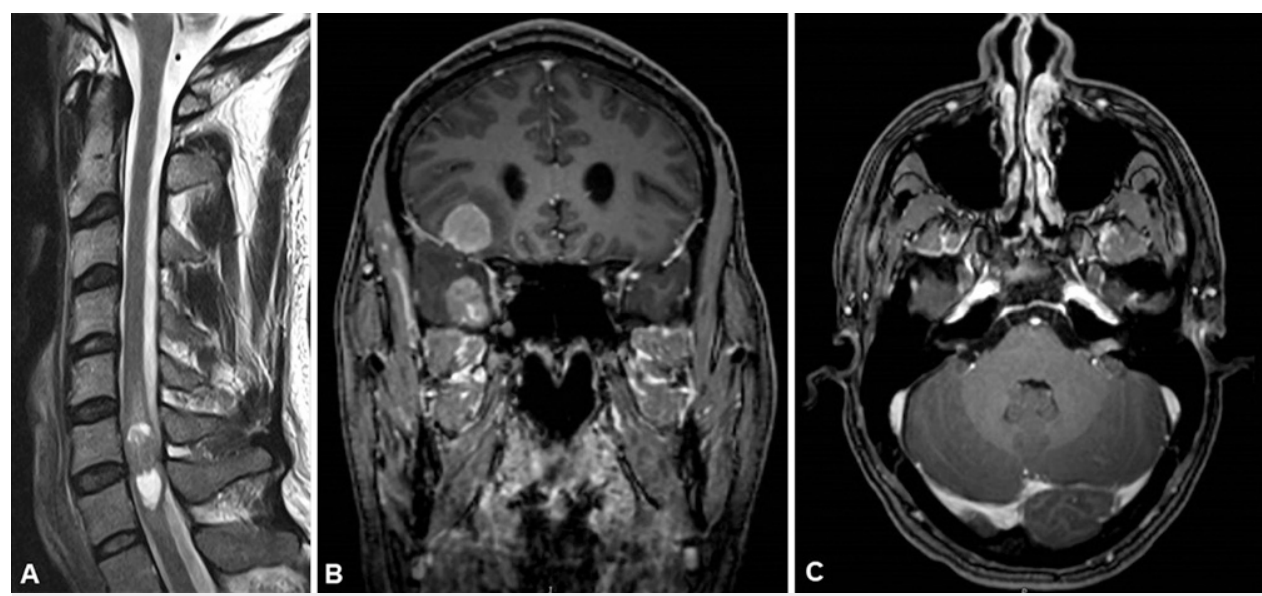

FIG. 1. Sagittal T2 MRI of the cervical spine showing cystic areas above and below a solid C6-C7 intramedullary lesion (A). MRI of the head showing enhancing masses in the right frontal and temporal lobes (B) and bilateral enhancing lesions in the internal auditory canal (C). 

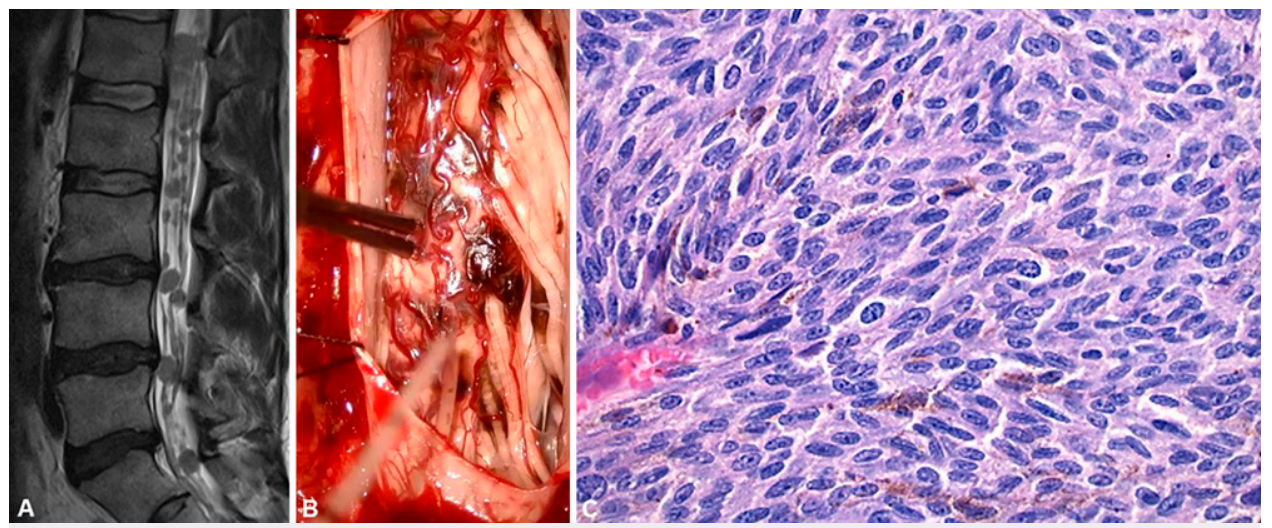

FIG. 2. A: MRI with sagittal T2-weighted image showing numerous extramedullary lesions along the cauda equina nerve roots. B: Intraoperative image of pigmented lesions on nerve roots, arachnoid open. C: Hematoxylin and eosin stain showing spindled cells with varying amounts of cytoplasmic melanin, occasional nucleoli, and longitudinal nuclear grooves (original magnification $\times 600$ ).

\section{Discussion}

\section{Observations}

We describe the case of a patient with imaging findings classic for neurofibromatosis type 2 but with biopsy findings showing melanocytic neoplasms with mild to moderate atypia and a mildly elevated proliferation index, which made the distinction between benign and malignant challenging. Furthermore, the disseminated nature of these tumors made it difficult to determine whether they arose from the meninges or represented metastases from an occult primary melanoma, a distinction that carries important prognostic and therapeutic implications.

\section{Lessons}

Primary melanocytic tumors of the CNS are a group of neoplasms ranging from benign to malignant and localized to diffuse that are thought to arise from leptomeningeal melanocytes. These melanocytes originate from neural crest cells, which differentiate into melanoblasts that migrate to mucosal surfaces, such as the aerodigestive and urogenital tracts, and to the inner ear, the uvea and leptomeninges during embryonic development. ${ }^{2}$ The CNS resident melanocytes differ from cutaneous melanocytes, sharing similarities with those of the uveal tract. This finding is further supported by the molecular similarities of both benign and malignant tumors that they give rise to.

Primary leptomeningeal melanocytic neoplasms can be broadly categorized as localized and diffuse. According to the 2016 edition of the World Health Organization (WHO) classification of tumors of the CNS, localized neoplasms may be designated as melanocytomas if they are benign or melanomas if they are malignant. Intermediate-grade lesions are also designated as melanocytomas. Diffuse lesions in the subarachnoid space that do not form macroscopic masses are termed melanocytosis and are regarded as benign. On histology, meningeal melanocytomas lack cytological atypia and mitosis. The term meningeal melanomatosis is used when primary CNS melanoma arises from and spreads throughout the subarachnoid and Virchow-Robin spaces and invades the CNS. ${ }^{1}$

\section{Meningeal Melanocytoma}

Melanocytomas are slow-growing, localized tumors with a peak incidence in the fifth decade of life with a slight female predominance (1.5:1). They most frequently arise in the cervical and thoracic spinal
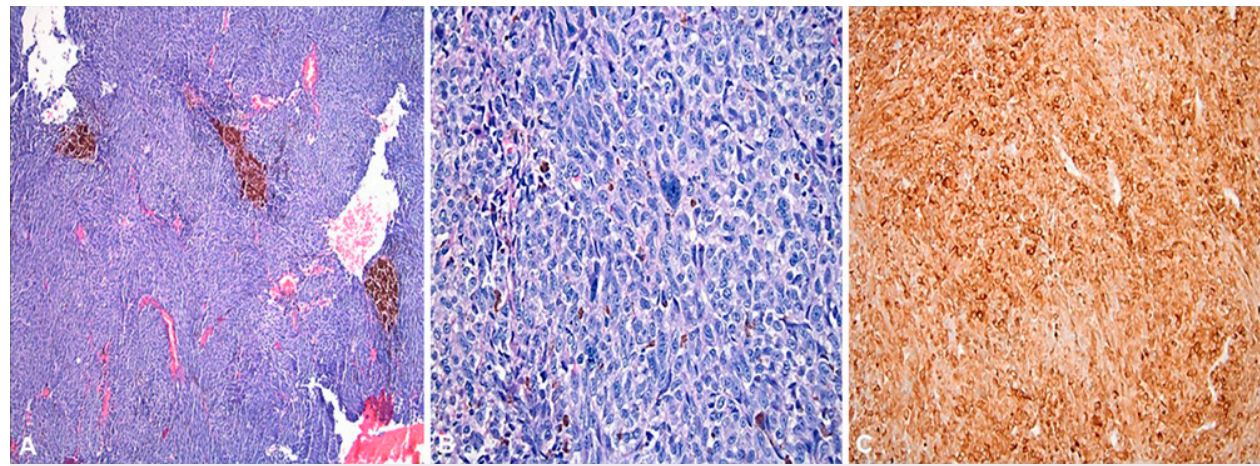

FIG. 3. A: Hematoxylin and eosin stain of the frontal mass revealing a hypercellular lesion with sheets of spindled cells, some with cytoplasmic melanin (original magnification $\times 100$ ). B: Hematoxylin and eosin stain of the temporal mass showing atypical ovoid cells with prominent nucleoli and occasional bizarre forms (original magnification $\times 400$ ). C: Lesional cells showing strong and diffuse immunopositivity with MART1 (original magnification $\times 200$ ). 
regions, the posterior cranial fossa, and Meckel's cave, likely because of the high density of melanocytes in these areas. In the spine, they are usually intradural and extramedullary and may be either dural based or associated with nerve roots or spinal foramina. Rarely, they may be intramedullary or paraspinal. ${ }^{1,2}$

Clinically, they usually present with compressive symptoms, with focal neurological signs depending on the tumor location. On MRI, the unique paramagnetic properties of melanin cause them to appear iso- to hyperintense on T1-weighted images and iso- or hypointense on T2-weighted images, with homogenous enhancement on postcontrast images. ${ }^{1,4}$

Macroscopically, these lesions may be black, reddish-brown, or blue in color, or they may be nonpigmented. Histological examination reveals them to be composed of well-differentiated spindle or ovoid melanocytes with minimal cytological atypia and varying amounts of cytoplasmic melanin, growing as tight nests or sheets. The nuclei are bean-shaped, and longitudinal grooves and micronucleoli may be appreciated. Importantly, these lesions should exhibit minimal to no mitotic activity (on average $<1$ mitosis per 10 high power fields), and the Ki-67 proliferative index is usually $<1 \%-2 \%$. Invasion of surrounding structures is not seen. The 2016 edition of the WHO classification of CNS tumors suggests that melanocytic tumors with bland cytological features, such as those of melanocytoma, but showing CNS invasion or elevated mitotic activity should be classified as intermediate-grade melanocytic neoplasms. ${ }^{1}$

Completely excised melanocytomas have a good prognosis, although local recurrence can occur with incomplete excision. ${ }^{1,5,6}$ Because of the low frequency of reported cases, the behavior of intermediate-grade lesions cannot be reliably predicted. ${ }^{1}$ Rarely, melanocytomas with aggressive behavior and malignant transformation have been reported. ${ }^{1}$

\section{Meningeal Melanoma}

Meningeal melanomas are the malignant counterpart to meningeal melanocytomas. They have an annual incidence of 0.5 per 10 million and may occur at any age, with a peak in the fifth decade of life. There is a slight male to female predominance. Meningeal melanoma may occur anywhere along the neuraxis, with a predilection for the spinal cord and posterior fossa.,

Like melanocytomas, they present with focal neurological symptoms depending on the tumor location. Imaging findings are similar to those of melanocytomas, although they may have T2 hyperintensity in the adjacent brain or spinal cord, which is indicative of vasogenic edema generated by rapid tumor growth. ${ }^{1}$

Histologically, these tumors resemble melanomas from other sites. The cells may be anaplastic, either epithelioid or spindled, and form fascicles, sheets, and nests with varying degrees of cytoplasmic melanin pigment. In some cases, large bizarre cells may be present with significant pleomorphism and large nucleoli. Other cases may be less pleomorphic. Typical and atypical mitotic figures are numerous, and there is often unequivocal invasion of the CNS parenchyma and coagulative necrosis.

The prognosis of primary meningeal melanoma is better than that of melanoma metastatic to leptomeninges, especially in the case of localized lesions in which complete resection is possible.

The main differential diagnosis of meningeal melanoma is metastatic melanoma, particularly from regressed or nonvisible lesions, such as acral melanoma, melanomas of the nail bed, or mucosal surfaces. A diagnosis of metastatic melanoma should be excluded by careful review of a patient's history, detailed physical examination, and ophthalmic evaluation and reexamination of any previously excised skin lesions. Another important entity to consider in the differential diagnosis of melanocytic neoplasms is the melanotic schwannoma. This is a variant of schwannoma composed of Schwann cells with intracytoplasmic melanosomes, which cause them to express melanocytic markers. These lesions may exhibit cytological atypia with hyperchromasia and macronucleoli and may be difficult to differentiate from melanocytic tumors. Reticulin staining or immunohistochemistry for basement membrane proteins (such as collagen IV) may be useful because staining will be nested in melanocytomas, whereas schwannomas tend to demonstrate pericellular staining. Other uncommon entities to consider in the differential diagnosis of melanocytic neoplasms include melanotic medulloblastoma, melanotic neuroectodermal tumor of infancy, pigmented glial or ependymal and choroid plexus tumors, teratomas, and paragangliomas. ${ }^{1,2}$ Rarely, meningiomas can have entrapped pigmented dendritic melanocytes and could mimic melanocytoma. ${ }^{7}$

\section{Diffuse Melanocytic Lesions}

Melanocytosis and melanomatosis are characterized by diffuse proliferation of benign or malignant melanocytes, respectively, along the leptomeninges, the latter exhibiting superficial invasion of the CNS parenchyma. Both occur primarily in children and are strongly linked to neurocutaneous melanosis, a rare phakomatosis characterized by the presence of large and/or multiple congenital melanocytic nevi of the skin with primary melanocytic lesions of the CNS. ${ }^{1,4}$

Melanocytosis may remain asymptomatic; however, the prognosis is poor once symptoms develop, even in the absence of malignant transformation. Symptoms may be secondary to hydrocephalus or local effects on CNS parenchyma, with neuropsychiatric symptoms and bowel, bladder, sensory, and motor dysfunction being most common. Melanomatosis, on the other hand, is a highly aggressive disease with a high mortality. ${ }^{1,4}$

\section{Molecular Features}

Primary leptomeningeal melanocytic neoplasms have a molecular profile that is distinct from cutaneous melanomas and more closely resembles that of uveal melanomas and blue nevi. Cutaneous melanomas most frequently harbor activating mutations of the BRAF gene (present in approximately $50 \%$ of cases), the most common mutation occurring at the hotspot BRAF V600E and accounting for $75 \%$ of cases. Other mutations include activating NRAS mutations found in $15 \%-20 \%$ of cases and mutations in the promoter region of the TERT gene. Interestingly, NRAS is seen in childhood melanoma and neurocutaneous melanosis, and acquisition of somatic mutations in NRAS in CNS melanocytes is a predisposing risk factor for primary melanoma of the CNS in children. ${ }^{8}$ Mucosal and acral melanomas (melanomas of non-hair-bearing skin) most frequently harbor activating mutations and/or increased copy numbers of the receptor tyrosine kinase KIT $(20 \%-30 \%)$ and NRAS (20\%), with BRAF and TERT promoter mutations being rare.

In contrast, uveal melanoma and blue nevi frequently harbor activating and mutually exclusive mutations of the GNAQ gene (approximately $45 \%$ and $83 \%$, respectively) or the GNA11 gene (32\% and 6.5\%, respectively). For both genes, these mutations occur at the hotspot codon 209 or more rarely codon $183(1 \%-3 \%){ }^{2}$ Currently, a clear association between mutation status and survival is lacking; however, uveal melanoma metastases tend to have a higher frequency of GNA11 mutations. ${ }^{5}$ 
GNAQ and GNA11 mutations are postulated to represent the driver mutations for uveal melanoma and are the main mutations seen in meningeal melanocytomas. Acquiring SF3B1 or EIF1AX mutations or inactivation of the $B A P 1$ gene is commonly seen with malignant transformation. BAP1 gene inactivation and SF3B1 and EIF1AX gene mutations are three mutually exclusive alterations. BAP1 mutations are typically associated with a worse prognosis, with $80 \%$ of metastatic uveal melanomas harboring BAP1 mutations. ${ }^{2}$ On the other hand, SF3B1 and EIF1AX mutations tend to occur in tumors that do not metastasize.

Similarly to uveal melanoma and blue nevi, both benign and malignant circumscribed CNS melanocytic lesions harbor activating mutations in either the GNAQ or GNA11 genes, also occurring at the hotspot codons 209 (most common) and 183. ${ }^{9-11}$ According to a review of previously published series, GNAQ mutations are estimated to occur in $39 \%$ of melanocytomas and $17 \%$ of melanomas, and GNA11 mutations are estimated to occur in $17 \%$ of melanocytomas and $29 \%$ of melanomas. ${ }^{2}$ Because of the limited number of primary leptomeningeal melanocytic lesions analyzed, other mutations are less well characterized.

In one study, 24 primary CNS melanocytic lesions were analyzed for the presence of SF3B1 and EIF1AX mutations in addition to loss of BAP1 by immunohistochemistry. ${ }^{12}$ All cases demonstrated intact BAP1 staining; however, $13 \%$ of cases harbored SF3B1 mutations and $21 \%$ harbored EIF1AX mutations, both being mutually exclusive. Interestingly, all cases harboring the SF3B1 mutation demonstrated aggressive behavior, with leptomeningeal seeding shortly after diagnosis, despite one case being diagnosed as a melanocytoma. The presence and prognosis of BAP1 mutations in meningeal melanomas remains to be characterized. One study of 19 primary CNS melanocytic tumors (all either melanocytomas or intermediate-grade lesions) detected a BAP1 inactivating mutation in one case, which later recurred. ${ }^{5}$ Furthermore, another reported case of BAP1-mutated meningeal melanoma occurred in a patient with a germline mutation in BAP1 and recurred after only a year. ${ }^{13}$

The same cohort of 19 cases of primary CNS melanocytic tumors was reanalyzed for hotspot mutations in the CYSLTR2 and PLCB4 genes, which are activating mutations that have been recently detected in GNAQ and GNA11 wild-type uveal melanomas. ${ }^{5,14}$ Further highlighting the similarities between uveal melanoma and meningeal melanocytic neoplasms, mutations in CYSLTR2 were identified in two of the samples analyzed, and mutations in PLCB4 were detected in one sample. These mutations were found to occur at the same hotspots previously identified for uveal melanoma and were mutually exclusive with GNAQ and GNA11 mutations.

In contrast to cutaneous melanoma, BRAF, KIT, and TERT promoter mutations are rare in primary CNS melanocytic neoplasms; if present, they should raise suspicion of a metastatic lesion. NRAS mutations, however, have been reported in both cutaneous and meningeal melanocytic lesions of children with neurocutaneous melanosis. These mutations are thought to occur before melanocytic migration as postzygotic somatic mutations. Supporting this notion, in the aforementioned series, one of the melanocytic tumors with an SF3B1 mutation was from a patient with neurocutaneous melanosis who also harbored an NRAS mutation. The melanocytic skin nevus from the same patient was found to harbor the same NRAS mutation; however, it did not have the SF3B1 mutation. ${ }^{9}$ The molecular similarities between primary CNS melanocytic neoplasms, uveal melanoma, and blue nevi are striking because these neoplasms all derive from nonepithelial melanocytes (blue nevi being intradermal). ${ }^{6}$
In summary, primary CNS melanocytic neoplasms represent a rare group of neoplasms, ranging from benign to malignant tumors. They may present as localized mass lesions or as diffuse proliferations involving leptomeninges, the latter of which is often associated with neurocutaneous melanosis syndrome. The diagnosis of these tumors can be challenging, as illustrated by this case, and requires integration of clinical and radiographic findings with histological appearance and molecular studies. This approach is especially required to determine whether the lesion represents a primary CNS lesion versus a metastatic melanoma, an important distinction because they differ both in prognosis and management. From a molecular standpoint, meningeal melanocytic lesions more closely resemble those of the uveal tract; however, further studies are required to determine the prognostic significance of SF3B1 and EIF1AX mutations as well as the prognosis of $B A P 1$ inactivating mutations.

\section{References}

1. Ohgaki H, Wiestler OD, Cavenee WK, et al. WHO Classification of Tumors of the Central Nervous System. International Agency for Research on Cancer; 2016.

2. Küsters-Vandevelde HVN, Küsters B, van Engen-van Grunsven ACH, Groenen PJTA, Wesseling P, Blokx WAM. Primary melanocytic tumors of the central nervous system: a review with focus on molecular aspects. Brain Pathol. 2015;25(2):209-226.

3. Virchow R. Pigment und diffuse Melanose der Arachnoides. Virchows Arch. 1859;16:180-182.

4. Liubinas SV, Maartens N, Drummond KJ. Primary melanocytic neoplasms of the central nervous system. J Clin Neurosci. 2010;17(10): $1227-1232$.

5. van de Nes J, Gessi M, Sucker A, Möller I, Stiller M, Horn S, et al. Targeted next generation sequencing reveals unique mutation profile of primary melanocytic tumors of the central nervous system. J Neurooncol. 2016;127(3):435-444.

6. Küsters-Vandevelde HVN, Klaasen A, Küsters B, et al. Activating mutations of the GNAQ gene: a frequent event in primary melanocytic neoplasms of the central nervous system. Acta Neuropathol. 2010;119(3):317-323.

7. Nestor SL, Perry A, Kurtkaya O, et al. Melanocytic colonization of a meningothelial meningioma: histopathological and ultrastructural findings with immunohistochemical and genetic correlation. Case report. Neurosurgery. 2003;53(1):211-215.

8. Pedersen M, Küsters-Vandevelde HVN, Viros A, et al. Primary melanoma of the CNS in children is driven by congenital expression of oncogenic NRAS in melanocytes. Cancer Discov. 2013;3(4): 458-469.

9. Cornejo KM, Hutchinson L, Cosar EF, et al. Is it a primary or metastatic melanocytic neoplasm of the central nervous system? A molecular based approach. Pathol Int. 2013;63(11): $559-564$.

10. Küsters-Vandevelde HV, van Engen-van Grunsven IACH, Coupland $\mathrm{SE}$, et al. Mutations in $\mathrm{g}$ protein encoding genes and chromosomal alterations in primary leptomeningeal melanocytic neoplasms. Pathol Oncol Res. 2015;21(2):439-447.

11. van de Nes J, Wrede K, Ringelstein A, Stiller M, Horn S, Sucker A, et al. Diagnosing a primary leptomeningeal melanoma by gene mutation signature. J Invest Dermatol. 2016;136(7):1526-1528.

12. Küsters-Vandevelde HVN, Creytens D, van Engen-van Grunsven $\mathrm{ACH}$, et al. SF3B1 and EIF1AX mutations occur in primary leptomeningeal melanocytic neoplasms: yet another similarity to uveal melanomas. Acta Neuropathol Commun. 2016;4:5.

13. de la Fouchardière $A$, Cabaret $O$, Pètre $\mathrm{J}$, et al. Primary leptomeningeal melanoma is part of the BAP1-related cancer syndrome. Acta Neuropathol. 2015;129(6):921-923. 
14. van de Nes JAP, Koelsche C, Gessi M, et al. Activating CYSLTR2 and PLCB4 mutations in primary leptomeningeal melanocytic tumors. J Invest Dermatol. 2017;137(9):2033-2035.

\section{Disclosures}

The authors report no conflict of interest concerning the materials or methods used in this study or the findings specified in this paper.

\section{Author Contributions}

Conception and design: Lang-Orsini, Wu, Madan, Arkun. Acquisition of data: Lang-Orsini, Wu, Kravtsova, Madan, Arkun. Analysis and interpretation of data: all authors. Drafting the article: Lang-Orsini, Wu, Heilman, Madan, Arkun. Critically revising the article: Lang-Orsini, Wu, Heilman, Kravtsova,
Madan, Arkun. Reviewed submitted version of manuscript: Lang-Orsini, Heilman, Arkun. Approved the final version of the manuscript on behalf of all authors: Lang-Orsini. Administrative/technical/material support: Heilman, Kravtsova. Study supervision: Heilman, Arkun.

\section{Supplemental Information}

Previous Presentations

Portions of this work were presented at the 96th Annual Meeting of the

American Association of Neuropathologists, Atlanta, GA, June 11-14, 2020.

\section{Correspondence}

Melanie Lang-Orsini: Massachusetts General Hospital, Boston, MA. mlang-orsini@mgh.harvard.edu. 